\title{
APPLICATION OF GaN PRESSURE GROWN CRYSTALS FOR EPITAXY OF GaN-BASED STRUCTURES
}

\section{GRZEGORY}

High Pressure Research Center, Polish Academy of Sciences Sokołowska 29/37, 01-142 Warsaw, Poland

\begin{abstract}
The results obtained with the use of the pressure grown GaN single crystalline substrates allow to draw the following conclusions important for the construction of In-free UV light emitting diodes and lasers and InGaN-based high power blue lasers: 1. The application of the pressure grown GaN single crystalline substrates allows to grow near dislocation free layer structures by both metal organic chemical vapor deposition and MBE. 2. The elimination of dislocations leads to highly efficient UV emission from $\mathrm{GaN}$ and GaN/AlGaN quantum wells which is impossible for strongly dislocated structures grown on sapphire. 3. At high excitations (i.e. in lasers) dislocations are effective nonradiative recombination centers also in the InGaN containing structures, therefore the elimination of these defects is crucial for better performance of blue lasers. 4. The analysis of microstructural and optical properties of the In GaN containing dislocation free structures shows that the main mechanisms of carrier localization in $\mathrm{InGaN}$ are not related with the $\mathrm{nm}$ scale compositional fluctuations in InGaN. In the paper, the optical and structural properties of the near dislocation free GaN-based structures leading to the above conclusions are discussed.
\end{abstract}

PACS numbers: 81.10.-h, 81.05.Dz, 73.61.Ey, 78.66.Fd

\section{Introduction}

$\mathrm{GaN}$ and its alloys with $\mathrm{Al}$ and In became recently the basic materials for short-wavelength optoelectronics. It was mainly due to their direct energy gaps covering the whole visible spectrum and the large part of the UV range $(6.2$, 3.4 , and $1.9 \mathrm{eV}$ for AlN, GaN, and InN respectively). At present, high brightness blue and green light emitting diodes (LEDs) and low power blue laser diodes (LDs) are commercially available [1]. On the other hand however, the development of GaN-based technology was and still is, strongly limited by difficulties in obtaining large, high quality crystals which could be used as substrates for epitaxial deposition of multilayer quantum structures necessary for devices. This is direct consequence of thermodynamical properties of $\mathrm{GaN}$ [2], in particular its melting 
conditions (about $2800 \mathrm{~K}$, and over $45 \mathrm{kbar} \mathrm{N}_{2}$ pressure) which are so extreme that the application of the common growth methods from stoichiometric liquids is technically impossible.

The spectacular development of GaN-based optoelectronic devices was possible due to elaboration of the two-step metal organic chemical vapor deposition (MOCVD) process for the growth of (GaAlIn)N epitaxial structures on highly mismatched sapphire substrates. In these structures dislocation densities are as high as $10^{8}-10^{10} \mathrm{~cm}^{-2}$ but nevertheless very efficient luminescence is possible if the structures contain InGaN [1]. It is suggested that this is due to strong localization of carriers in deep potential wells caused by compositional fluctuation in InGaN alloys, which eliminates interaction of carriers with dislocations. However the size and the nature of these fluctuations were not precisely defined. For structures without indium, dislocations acting as the nonradiative recombination centers quench the luminescence and the construction of the efficient light emitting devices is at present impossible. In contrast to the InGaN based LEDs, for the LDs dislocations limit the device lifetime which is the most important obstacle in construction of high power blue lasers.

Gallium nitride crystals grown at high pressure of $\mathrm{N}_{2}$ give possibility to grow near dislocation free epitaxial layers and structures on both Ga-polar and N-polar [0001] surfaces of the substrate. It allows to estimate the influence of dislocations on the physical properties of the material as well as on the performance of GaN-based devices. Dislocations however are not the only defects lowering optical quality of the material. Each growth technique introduces its own set of structural and chemical defects related to the particular growth mechanisms and conditions. Even materials grown in the same run on near dislocation free substrates can differ substantially if the substrates are of different polarity or misorientation. Therefore one has to be very careful with drawing conclusions evaluating the role of dislocations on the properties of the nitride structures.

In the following sections, $\mathrm{GaN}$ crystals grown under pressure will be shortly characterized. Then some examples of dislocation free epitaxial GaN based structures will be analyzed to demonstrate the role of dislocations in both In-free and In-containing layers.

\section{Crystals}

\subsection{Physical properties}

The GaN crystals described here are grown. (i.e. [2]) at $\mathrm{N}_{2}$ pressure of $10-20 \mathrm{kbar}$, at temperatures $1500-1600^{\circ} \mathrm{C}$, from solutions of atomic nitrogen in liquid $\mathrm{Ga}$ (high nitrogen pressure solution (HNPS) method). The synthesis of GaN from its constituents is possible due to dissociative chemisorption of $\mathrm{N}_{2}$ molecule on the $\mathrm{Ga}$ surface [3]. Nitrogen molecule dissociates -on the Ga surface only if it overcomes the potential barrier of about $4 \mathrm{eV}$. For oxygen interacting with $\mathrm{Ga}$ there is no potential barrier for dissociation and therefore even traces of this impurity in the growth system are source of the unintentional oxygen doping of $\mathrm{GaN}$. Therefore the crystals are strongly $n$-type with free electron concentration of about $5 \times 10^{19} \mathrm{~cm}^{-3}$ (metallic conductivity) and mobility of about $60 \mathrm{~cm}^{2} /(\mathrm{V} \mathrm{s})$ [4]. 
These free carriers can be fully eliminated by $\mathrm{Mg}$ acceptor added into the growth solution. Then the resistivity of the crystals becomes as high as $10^{4}-10^{6} \Omega \mathrm{cm}$ at $300 \mathrm{~K}$. Usually the GaN:Mg crystals become $p$-type with an activation energy of $150 \mathrm{meV}$, at temperatures slightly exceeding $300 \mathrm{~K}$.

The presence of native point defects in the crystals has been checked by positron annihilation measurements [5]. High concentration of Ga vacancies $V_{\mathrm{Ga}}$ has been found in the conductive crystals in contrast to the $\mathrm{Mg}$-doped samples where no Ga-vacancies have been observed. This agreed with theoretical prediction that the formation energy of $V_{G a}$ decreases with the increase in the Fermi level energy [6] suggesting that the creation of these defects is thermodynamically controlled. The difference in the photoluminescence (PL) spectra of the conductive (strong yellow emission) and $\mathrm{Mg}$-doped crystals (no yellow emission, blue $\mathrm{Mg}$-related signal) supported that $\mathrm{V}_{\mathrm{Ga}}$ is involved in yellow luminescence in $\mathrm{GaN}$.

Quite different picture is observed for doping with beryllium [7]. The GaN:Be crystals are also highly resistive with the constant activation energy of $1.46 \mathrm{eV}$ at temperatures up to $1000 \mathrm{~K}$. But their PL spectra are dominated by a very strong yellow luminescence and they contain a lot of gallium vacancies [8] exactly like highly conductive crystals grown without an intentional doping. Therefore it is very probable that these crystals are $n$-type during high temperature growth and become semi-insulating only during cooling down. Such behavior can be related to the two possible configurations of $\mathrm{Be}$ atoms in $\mathrm{GaN}$ lattice $(\mathrm{Be} \mathrm{Ga}$ - acceptor and $\mathrm{Be}_{\mathrm{i}}$ - donor) [9] and their redistribution in function of temperature.

\subsection{Morphology and size}

The GaN crystals grown by the HNPS method are hexagonal platelets. This habit does not change for solutions containing $\mathrm{Mg}$ or $\mathrm{Be}$. The average size of crystals grown without an intentional seeding scales with the diameter of the high pressure reactor as shown in Fig. 1. Better control of the size and distribution of the growing crystals can be achieved by the intentional seeding. The seeded growth into $\{11-20\}$ directions gives good results in terms of growth stability and reproducibility of the processes.

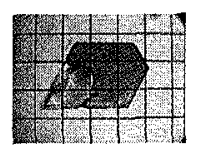

1
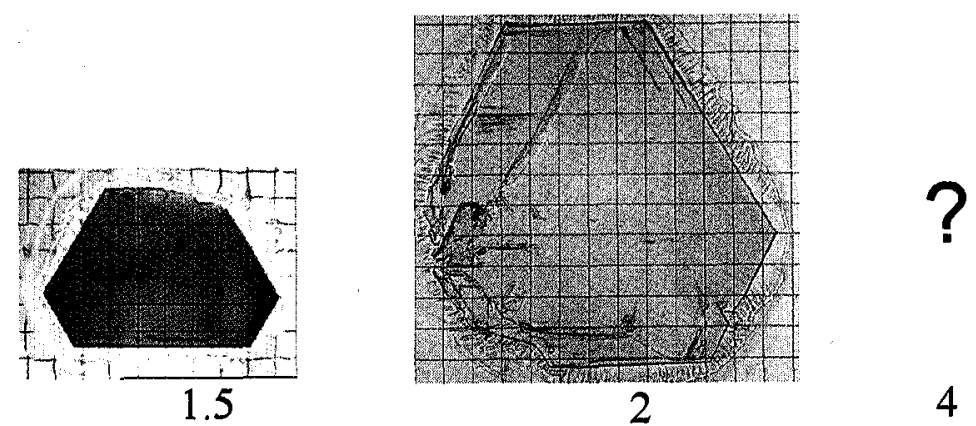

4

Fig. 1. GaN crystals grown at high pressure chambers of different size. The numbers are proportional to the diameters of the chambers. 


\subsection{Dislocation density}

The structure of the pressure grown GaN crystals has been studied by X-ray diffraction (XRD) [10], transmission electron microscopy (TEM) [11, 12], defect selective etching (DSE) [13] and atomic force microscopy (AFM) of the homoepitaxial layers [14].

All these methods indicate that the dislocation density in the crystals is very low. From large area DSE experiments it follows that the crystals contain less that $10^{2}$ dislocations per $\mathrm{cm}^{2}$. Figure 2a shows large area AFM scan of the GaN homoepitaxial layer grown by MOCVD in High Pressure Research Center [14]. The atomic step flow is not perturbed by dislocations [16] as it is for $\mathrm{GaN}$ layers containing threading dislocations (Fig. $2 \mathrm{~b}$ ).

The cleavage planes of the crystals are perpendicular to $\{10-10\}$ crystallographic directions.
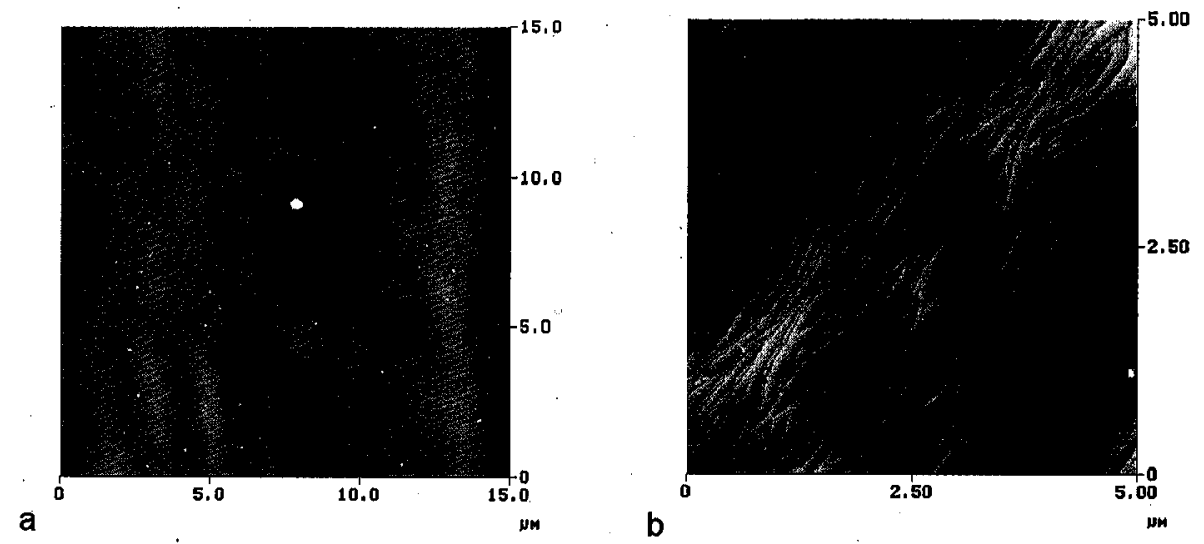

Fig. 2. Surface morphology of GaN epitaxial layers deposited by MOCVD [15] on different substrates: (a) GaN pressure grown crystal, (b) sapphire.

\section{GaN homoepitaxy on GaN pressure grown substrates}

If the surface preparation and the conditions of epitaxial growth are right, the structure of GaN homoepitaxial layers in terms of dislocation density, follows the structure of the GaN substrates. Therefore the layers are also near dislocation free and their optical properties depend mostly on the point defects incorporated during the epitaxial growth. It is a function of the purity of the growth system, growth conditions, and orientation of the substrate. Due to the lack of strains and the high degree of homogeneity of the homoepitaxial GaN, the exciton related peaks in low temperature (LT) PL spectra are usually very narrow. The FWHM of the bound exciton lines less than $0.5 \mathrm{meV}$ is observed for layers grown by both MOCVD (i.e. $[17 ; 18]$ ) and MBE (i.e. $[19,20]$ ) on the Ga-polar surface of the GaN substrates. For layers deposited on the N-polar surfaces the spectra are usually much wider due to enhanced incorporation of unintentional impurities on this chemically active side of GaN crystal. This can be suppressed by the use of surfaces 
vicinal to the (000-1) as it was shown by Zauner et al. [21] who obtained narrow excitonic spectra for GaN layers grown on misoriented GaN N-polar surface.

For $\mathrm{GaN}$ layers grown by MBE with $\mathrm{NH}_{3}$ nitrogen source, it was shown [20] that the band edge PL integrated intensity at room temperature (RT) for the homoepitaxial $\mathrm{GaN}$ is about 60 times larger than for $\mathrm{GaN}$ grown on sapphire at the same growth conditions. Also the decay of the PL in these homoepitaxial layers is much slower than for heteroepitaxial GaN [22]. This indicates a substantial suppression of the nonradiative recombination by elimination of threading dislocations from the layers. This opens a possibility for UV In-free light emitting devices.

The PL decay has been also studied at high excitations [23] for GaN layers grown by MOCVD on sapphire and pressure grown GaN substrates. The decay time (at RT) of 450 ps measured for homoepitaxial material was 5 times higher than for corresponding heteroepitaxial layer grown at the same conditions.

The optically pumped LT stimulated emission [24] experiments confirmed that the near dislocation free $\mathrm{GaN}$ is much more efficient source of light than $\mathrm{GaN}$ containing dislocations. The stimulated emission in GaN homoepitaxial layer grown by MOCVD started at the excitation power density lower than $1 \mathrm{MW} / \mathrm{cm}^{2}$ whereas for $\mathrm{GaN}$ grown on $\mathrm{SiC}$ [25] only at $10 \mathrm{MW} / \mathrm{cm}^{2}$. The stimulated emission peak for the homoepitaxial layer was as narrow as about $5 \mathrm{meV}$ (about $40 \mathrm{meV}$ for $\mathrm{GaN} / \mathrm{SiC}$ ).

All the results mentioned above show that dislocations are very efficient nonradiative recombination centers in $\mathrm{GaN}$ especially at high excitations.

\section{Structures and devices}

\subsection{Problems}

As it was mentioned in the introduction, there are also "defects" increasing the optical efficiency of nitrides. It is believed, as it was suggested in [1], that these "defects" are the short-range compositional fluctuations in InGaN alloys leading to localization of carriers and therefore to limitation of the influence of dislocations as the nonradiative recombination centers. In this model, the influence of dislocations on the optical efficiency of InGaN can be eliminated if the distance between dislocations exceeds the size of the potential fluctuations. However, for high injection currents (i.e. in LDs), the potential minima are too shallow to contain all the injected carriers and therefore for lasers, the reduction of dislocation density in the material is much more important than for LEDs. The above model explains in a consistent way the following characteristics of Nakamura's (the unquestionable leader in the field of GaN-based technology and optoelectronics) devices:

- The nitride LEDs containing In in their active structures are much more efficient than LEDs with pure GaN active layers (not alloyed with In).

- InGaN LEDs efficiency does not depend on dislocation density (if $\mathrm{Al}_{2} \mathrm{O}_{3}$ or epitaxial lateral over-growth (ELOG) substrates are used) in contrast to the efficiency of In-free UV diodes.

- At high injection currents, the high dislocation density decreases the efficiency even for InGaN LEDs. 
Therefore, one can expect that the elimination of dislocations from the structures should lead to:

- High efficiency In-free UV LEDs.

- Higher efficiency and higher power of both UV and visible laser diodes than it is possible with dislocated structures.

In the following both In-free and In-containing structures are considered and the results supporting the above expectations are presented. Additionally, for the In-containing structures a special attention is paid to the results contributing into understanding of the physical phenomena responsible for highly efficient luminescence from InGaN.

\subsection{GaN/AlGaN - structures without indium}

The growth of GaN/AlGaN structures on GaN pressure grown crystals was studied mainly by MBE $[12,20,26,27]$. Near dislocation free GaN/AlGaN multiquantum wells and structures were grown on the N-polar surfaces of GaN crystals by RF plasma assisted MBE (PA MBE) $[12,27]$ and on the Ga-polar surfaces by MBE with $\mathrm{NH}_{3}$ nitrogen source (R MBE) $[20,26]$. As a rule, the optical properties of structures without dislocations were much better than for similar structures grown at the same conditions, on sapphire.

For example, in Fig. 3 the integrated intensities of the PL coming from similar structures (8 ML GaN single quantum well (SQW) with the $\mathrm{Al}_{0.1} \mathrm{Ga}_{0.9} \mathrm{~N}$ $50 \mathrm{~nm}$ barriers) grown by $\mathrm{R}$ MBE [26] on sapphire and bulk pressure grown GaN substrates are compared. It is shown that for the structure deposited on GaN, the $\mathrm{PL}$ is much stronger especially at RT because its intensity starts to decrease with temperature only at about $100 \mathrm{~K}$ mainly due to the thermal escape of carriers

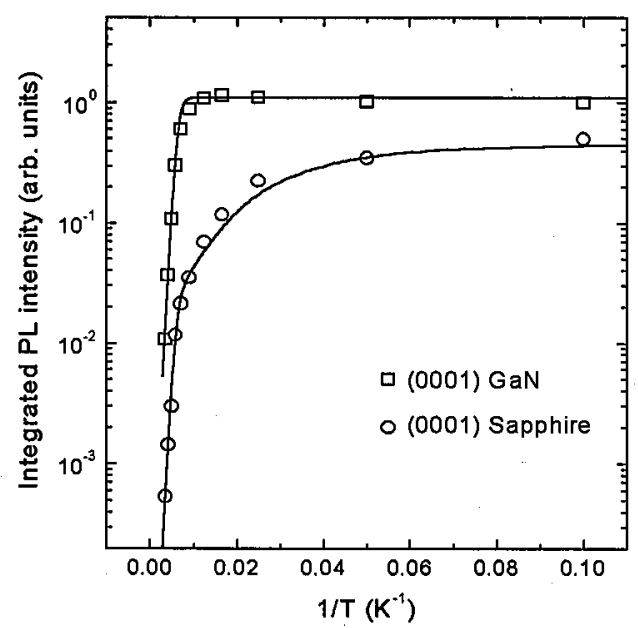

Fig. 3. Temperature dependence of the integrated PL intensity of homoepitaxial (squares) and heteroepitaxial (circles) $\mathrm{GaN} / \mathrm{Al}_{0.1} \mathrm{Ga}_{0.9} \mathrm{~N}$ QWs. 
from the quantum well towards the AlGaN barriers, as was observed for classical III-V QW heterostructures with dislocation densities lower than $10^{3} \mathrm{~cm}^{-2}$ [28]. For heteroepitaxial structure, the presence of dislocations strongly influences the nonradiative recombination processes. The PL intensity starts to decrease at much lower temperatures due to delocalization of excitons and their further interaction with dislocations.

It is especially important for devices, that further increase in the emission efficiency can be achieved for dislocation free structures by increasing the $\mathrm{Al}$ content in the barriers. This opens the possibility for construction of highly efficient UV LEDs and lasers.

The semi-insulating GaN:Mg substrates have been used for growth GaN/AlGaN heterostructures with two-dimensional electron gas (2DEG) by RMBE [29]. The Hall mobility for 2DEG as high as $60-100 \mathrm{~cm}^{2} /(\mathrm{V} \mathrm{s})$ (the highest reported for $\mathrm{GaN}$ ), at $1.5 \mathrm{~K}$ has been measured.

The Shubnikov-de Haas oscillations starting at $1.8 \mathrm{~T}$ and well defined quantum Hall effect (QHE) indicated high quality of the MBE grown heterostructure. From the analysis of the scattering processes it was suggested that in such near dislocation free structures the ionized impurity scattering is the dominant low temperature mobility limiting mechanism.

\subsection{In GaN containing structures}

The InGaN near dislocation free epitaxial layers and quantum wells were grown on the pressure grown crystals mainly by MOCVD [15]. Also the standard $470 \mathrm{~nm}$ LED structure has been grown by Nakamura [30] on the Unipress pressure

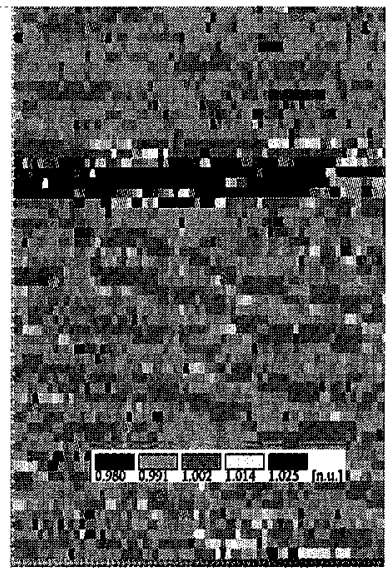

a

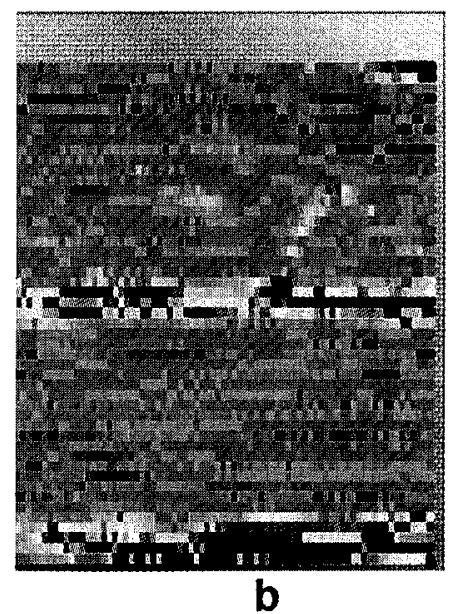

b

Fig. 4. High resolution TEM tetragonal distortion analysis [31] of the GaN/InGaN (15\% of In) quantum wells with different microstructures: (a) no compositional fluctuations in InGaN layer, (b) large compositional fluctuations in InGaN layer (n.u. normalized units (with respect to undistorted GaN crystal)). 
grown crystal. The compositional homogeneity of these materials was extensively studied by Albrecht et al. [31] using TEM and cathodoluminescence (CL) methods. It was shown that the InGaN microstructure is very sensitive to the growth conditions (temperature, growth rate, microscopic growth mode) and different InGaN microstructure can be observed even for samples of the same average In content. For example, for InGaN QWs with 15\% of In, the high resolution TEM (HRTEM) tetragonal distortion analysis has shown either perfectly homogeneous distribution of In or 8-20\% fluctuations of the In content with dimensions of few nm - Fig. 4. Nevertheless, the optical properties of these QWs, with very different microstructures were strikingly similar. It is shown in Fig. 5 where the cathodoluminescence spectra coming from these QWs are compared. The figure shows that the main CL emission lines coming from $\mathrm{QW}$ grown on GaN substrates and having large (8-20\%) In compositional fluctuations on a nanometer scale, have very similar energy position and the FWHM as the line from the homogeneous QW with the same average In fraction in the well. Figure 6 compares the electroluminescence (EL) spectra from LEDs with $\operatorname{In}_{0.15} \mathrm{Ga}_{0.85} \mathrm{~N} \mathrm{SQW}$ grown on different substrates. Again the spectra are very similar independently of the substrate used.

Therefore, it can be suggested that the nm scale compositional fluctuations often observed in InGaN, are not responsible for the high efficiency InGaN based LEDs which was the one of the most commonly accepted explanations. This suggestion is consistent with the observation of Prystawko and Leszczyński [15] that the PL peaks energy position for InGaN QWs grown on various substrates (sap-

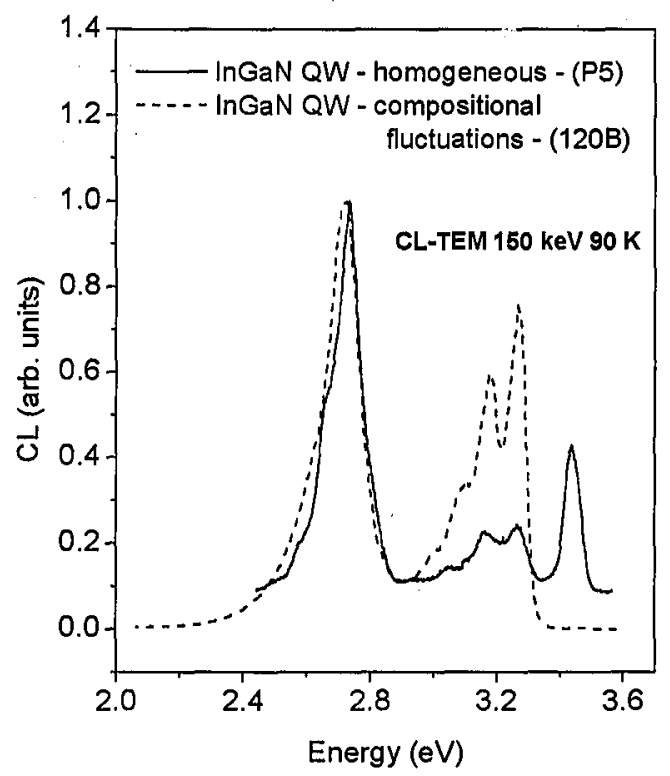

Fig. 5. Cathodoluminescence spectra coming from InGaN MQW grown on GaN substrate with large fluctuations of In content and from QW with homogeneous In distribution in InGaN. The average In fraction is the same in both structures. 


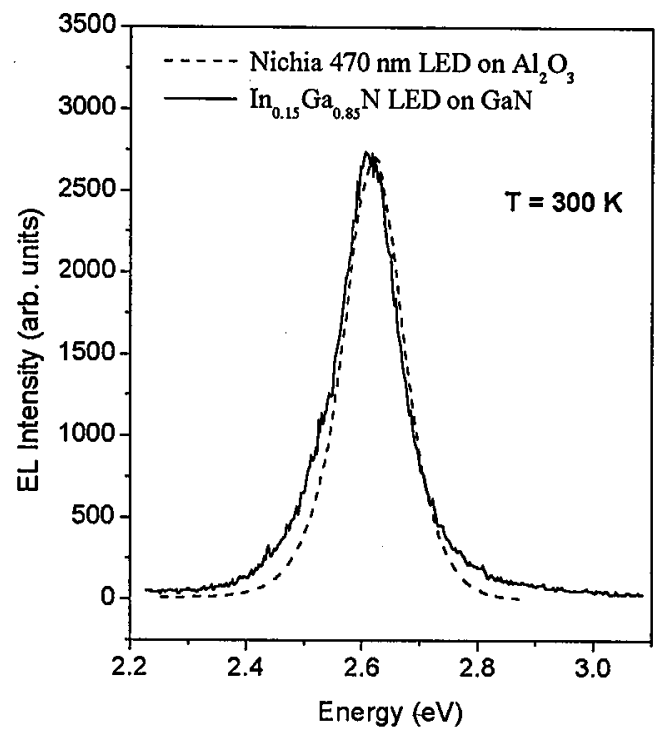

Fig. 6. Electroluminescence spectra from LEDs with InGaN SQW grown on different substrates. The average In fraction is the same in both structures.

phire, $\mathrm{SiC}$ and $\mathrm{GaN}$ ) depend only on the average In content (measured by X-ray and Rutherford backscattering (RBS)), not on dislocation density.

The last observation and the dependence obtained is in full agreement with the data of O'Donnell et al. [32] who found a linear relationship between PL band peak energy and measured In fraction in InGaN (for In content ranging from 0 to $40 \%$ ). The data allowed to conclude that localization in InGaN is due to non-random distribution of In atoms in InGaN in the atomic scale.

Therefore, it seems that indeed:

- The strong localization of carriers in InGaN are not due to detectable compositional fluctuations because the emission from the homogeneous InGaN is the same as from $\mathrm{InGaN}$ with the large fluctuations detected, with similar average In content.

- The presence of centers localizing the carriers is the intrinsic property of InGaN on the atomic level. The additional, very important support for this statement is that the emission peaks coming from InGaN of the same average composition are relatively broad (few tenths of $\mathrm{eV}$ even for homogeneous, dislocation free material) and of the same FWHM independently of the presence of the In content fluctuations.

The pressure grown GaN crystal have been tested by Nakamura [30] as substrate for the InGaN $405 \mathrm{~nm}$ laser. The $\mathrm{cw} 30 \mathrm{~mW}$ devices with lifetime exceeding $3000 \mathrm{~h}$ were constructed. The $30 \mathrm{~mW}$ power has been achieved at current of $62 \mathrm{~mA}$. For typical InGaN MQW LDs [1], at current of $62 \mathrm{~mA}$ the output power is about $15 \mathrm{~mW}$. This increase in the efficiency confirms that dislocations are the main limiting factors for high power GaN-based lasers. 


\section{Conclusions}

Both conductive and semi-insulating GaN pressure grown crystals are excellent substrates for epitaxial growth of near dislocation free nitride structures. They allow to limit dislocation densities to very low values (lower than $10^{2} \mathrm{~cm}^{-2}$ ) and therefore to eliminate important nonradiative recombination centers in both In-free and In-containing structures. It is of crucial importance for efficient UV emitting devices and for both UV and visible GaN-based high power lasers.

The analysis of microstructural and optical properties of the InGaN containing dislocation free structures allows to conclude that the main mechanisms of carrier localization in In GaN are not related with the $\mathrm{nm}$ scale compositional fluctuations in InGaN.

\section{References}

[1] S. Nakamura, IEEE Trans. Electron. E83-C, 529 (2000).

[2] I. Grzegory, J. Jun, M. Boćkowski, S. Krukowski, M. Wróblewski, B. Eucznik, S. Porowski, J. Phys. Chem. Solids 56, 639 (1995).

[3] S. Krukowski, Z. Romanowski, I. Grzegory, S. Porowski, J. Cryst. Growth 189/190, 159 (1999).

[4] S. Porowski, M. Boćkowski, B. Eucznik, I. Grzegory, M. Wróblewski, H. Teisseyre, M. Leszczyński, E. Litwin-Staszewska, T. Suski, P. Trautman, K. Pakuła, J.M. Baranowski, Acta Phys. Pol. A 92, 958 (1997).

[5] K. Saarinen, T. Laine, S. Kuisma, P. Hautojarvi, L. Dobrzyński, J.M. Baranowski, K. Pakuła, R. Stępniewski, M. Wojdak, A. Wysmołek, T. Suski, M. Leszczyński, I. Grzegory, S. Porowski, Phys. Rev. Lett. 79, 3030 (1997).

[6] J. Neugebauer, C.G. Van de Walle, Phys. Rev. B 50, 8067 (1994).

[7] M. Boćkowski, T. Suski, E. Litwin-Staszewska, P. Perlin, to be presented on EGW-4, Nottingham 2000.

[8] K. Saarinen, to be presented on EGW-4, Nottingham 2000.

[9] F. Bernardini, V. Fiorentini, Appl. Phys. Lett. 70, 2990 (1997).

[10] M. Leszczyński, I. Grzegory, H. Teisseyre, T. Suski, M. Boćkowski, J. Jun, J.M. Baranowski, S. Porowski, J. Domagała, J. Cryst. Growth 169, 235 (1996).

[11] Z. Lilienthal-Weber, S. Ruvimov, Ch. Kisielowski, Y. Chen, W. Swider, J. Washborn, N. Newman, A. Gassmann, X. Liu, L. Schloss, E.R. Weber, I. Grzegory, M. Boćkowski, J. Jun, T. Suski, K. Pakuła, J. Baranowski, S. Porowski, H. Amano, I. Akasaki, Mater. Res. Soc. Symp. Proc. 395, 351 (1996).

[12] S.H. Christiansen, M. Albrecht, H.P. Strunk, C.T. Foxon, D. Korakakis, I. Grzegory, S. Porowski, Physica Status Solidi A 176, 285 (1999).

[13] J.L. Weyher, P.D. Brown, J.L. Rouviere, T. Wosiński, A.R.A. Zauner, I. Grzegory, J. Cryst. Growth 210, 151 (2000).

[14] M. Leszczyński, P. Prystawko, G. Nowak, private communication.

[15] P. Prystawko, M. Leszczyński, to be published.

[16] H. Marchand, J.P. Ibbetson, P.T. Fini, X.H. Wu, S. Keller, S.P. DenBaars, J.S. Speck, U.K. Mishra, MRS Internet J. Nitride Semicond. Res. 4S1, G4.5 (1999).

[17] J.M. Baranowski, Mater. Res. Soc. Symp. Proc. 449, 393 (1997). 
[18] C. Kirchner, V. Schwegler, F. Eberhard, M. Karnp, K.J. Ebeling, K. Kornitzer, T. Ebner, K. Thonke, R. Sauer, P. Prystawko, M. Leszczyński, I. Grzegory, S. Porowski, Appl. Phys. Lett. 75, 1098 (1999).

[19] H. Teisseyre, G. Nowak, M. Leszczyński, I. Grzegory, M. Boćkowski, S. Krukowski, S. Porowski, M. Mayer, A. Pelzmann, M. Kamp, K.J. Ebeling, G. Karczewski, MRS Internet J. Nitride Semicond. Res. 1, 13 (1996).

[20] N. Grandjean, B. Damilano, J. Massies, G. Neu, M. Teissere, I. Grzegory, S. Porowski, M. Gallart, P. Lefebvre, B. Gil, M. Albrecht, J. Appl. Phys., accepted for publication.

[21] A.R.A. Zauner, J.L. Weyher, M. Plomb, V. Kirilyuk, I. Grzegory, W.J.P. van Enckevort, J.J. Schermer, P.R. Hageman, P.K. Larsen, J. Cryst. Growth 210, 435 (1900).

[22] T. Taliercio, M. Gallart, P. Lefebvre, A. Morel, B. Gil, J. Allegre, N. Grandjean, J. Massies, I. Grzegory, S. Porowski, to be published.

[23] A. Zakauskas, M. Leszczyński, P. Perlin, T. Suski, S. Porowski, to be presented on EGW-4, Nottingham 2000.

[24] J.Chr. Holst, A. Hoffman, M. Leszczyński, S. Porowski, to be published.

[25] J.Chr. Holst, L. Eckey, A. Hoffman, I. Broser, H. Amano, I. Akasaki, MRS Internet J. Nitride Semicond. Res. 2, 25 (1997).

[26] N. Grandjean, J. Massies, I. Grzegory, S. Porowski, Appl. Phys. Lett., accepted for publication.

[27] C.T. Foxon, C.S. Davis, S.V. Novikov, O.H. Hughes, T.S. Cheng, D. Korakakis, N.J. Jeffs, I. Grzegory, S. Porowski, Physica Status Solidi A 176, 723 (1999).

[28] J.D. Lambkin, D.J. Dunstan, K.P. Homewood, L.K. Howard, M.T. Emeny, Appl. Phys. Lett. 57, 1986 (1990).

[29] E. Frayssinet, W. Knap, P. Lorenzini, N. Grandjean, J. Massies, C. Skierbiszewski, T. Suski, I. Grzegory, S. Porowski, G. Simin, X. Hu, M. Asif Khan, M. Shur, R. Gaska, D. Maude, to be published.

[30] S. Nakamura, private communication.

[31] M. Albrecht, V. Grillo, H.P. Strunk, P. Prystawko, M. Leszczyński, I. Grzegory, S. Porowski, presented on the E-MRS Meeting, Strasbourg 2000.

[32] K.P. O'Donnell, R.W. Martin, C. Trager-Cowan, M.E. White, K. Esona, C. Deatcher, P.G. Middleton, K. Jacobs, W. Van der Stricht, C. Merlet, B. Gil, A. Vantomme, J.F.W. Mosselmans, presented on the E-MRS Meeting, Strasbourg 2000 , to be published in the Conference Proceedings. 